\title{
ASSOCIATION BETWEEN TEMPERATE DISTRIBUTIONS AND ANALYTICAL FUNCTIONS IN THE CONTEXT OF WAVE-FRONT SETS
}

\author{
KAROLINE JOHANSSON
}

\begin{abstract}
Let $\mathscr{B}$ be a translation invariant Banach function space (BF-space). In this paper we prove that every temperate distribution $f$ can be associated with a function $F$ analytic in the convex tube $\Omega=\left\{z \in \mathbf{C}^{d} ;|\operatorname{Im} z|<1\right\}$ such that the wave-front set of $f$ of Fourier BF-space types in intersection with $\mathbf{R}^{d} \times S^{d-1}$ consists of the points $(x, \xi)$ such that $F$ does not belong to the Fourier BF-space at $x-i \xi$.
\end{abstract}

\section{INTRODUCTION}

Wave-front sets of Fourier Banach function types where introduced by Coriasco, Johansson and Toft in [1]. Roughly speaking, the wavefront set of Fourier Banach function type, $\mathrm{WF}_{\mathscr{F} \mathscr{B}}(f)$, of a distribution $f$, consists of all pairs $\left(x_{0}, \xi_{0}\right)$ such that no localization of the distribution $f$ at $x_{0}$ belongs to $\mathscr{F} \mathscr{B}$ in the direction $\xi_{0}$. Several properties of classical wave-front sets (with respect to smoothness) can be found in Hörmander [12. One of these are mapping properties for pseudodifferential operators (with smooth symbols) on wave-front sets which were generalized to Fourier Lebesgue type by Pilipovic, Teofanov and Toft in [14]. These properties were also proved to hold for wave-front sets of Fourier Banach function types. (Cf. Coriasco, Johansson and Toft [1.)

In this paper we consider another property of wave-front sets concerning association between a temperate distribution and an analytic function, which was proved for classical wave-front sets by Hörmander in [12]. More precisely, Hörmander showed that every temperate distribution $f$ can be associated with a function $F$ analytic in the convex tube $\left\{z \in \mathbf{C}^{d} ;|\operatorname{Im} z|<1\right\}$ such that

$$
f=\int_{|\xi|=1} F(\cdot+i \xi) d \xi
$$

Date: November 10, 2018.

2000 Mathematics Subject Classification. 35A18.

Key words and phrases. Wave-front, Fourier, Banach function space. 
and

$$
\left(\mathbf{R}^{d} \times S^{d-1}\right) \cap \mathrm{WF}_{L}(f)
$$$$
=\left\{(x, \xi) ;|\xi|=1, F \text { is not in } C^{L} \text { at } x-i \xi\right\} .
$$

Here $\mathrm{WF}_{L}(f)$ is the wave-front set with respect to a class of smooth functions $C^{L}$. (Cf. Section 8.4 in Hörmander [12].)

In this paper we generalize this result to wave-front sets of Fourier Banach function types. We show that for every temperate distribution $f$ there exists a function $F$ with the properties given before, satisfying (0.1) and such that

$$
\begin{aligned}
\left(\mathbf{R}^{d} \times S^{d-1}\right) \cap \mathrm{WF}_{\mathscr{F} \mathscr{B}}(f) & \\
& =\{(x, \xi) ;|\xi|=1, F \text { is not in } \mathscr{F} \mathscr{B} \text { at } x-i \xi\} .
\end{aligned}
$$

Since every Lebesgue space is a Banach function space we get by choosing $\mathscr{B}=L^{p}$ that the analogous result for wave-front sets of Fourier Lebesgue types is contained in (0.2) as a special case.

As shown later on in this paper, analogous results hold also for the weighted cases as well as inf types and modulation space types of wavefront sets. The latter is a direct consequence of the identification of wave-front sets of Fourier BF-spaces types with wave-front sets of modulation space types.

The modulation spaces were introduced by Feichtinger in [2], and the theory was developed in [4,6, 9]. The modulation space $M(\omega, \mathscr{B})$, where $\omega$ is an appropriate weight function (or time-frequency shift) on phase space $\mathbf{R}^{2 d}$, appears as the set of temperate (ultra-)distributions whose short-time Fourier transform belong to the weighted Banach space $\mathscr{B}(\omega)$. This family of modulation spaces contains the (classical) modulation spaces $M_{(\omega)}^{p, q}\left(\mathbf{R}^{2 d}\right)$ as well as the space $W_{(\omega)}^{p, q}\left(\mathbf{R}^{2 d}\right)$ related to the Wiener amalgam spaces. In fact, these spaces which occur frequently in the time-frequency community are obtained by choosing $\mathscr{B}=L_{1}^{p, q}\left(\mathbf{R}^{2 d}\right)$ or $\mathscr{B}=L_{2}^{p, q}\left(\mathbf{R}^{2 d}\right)$ (see Remark 6.1 in [1]).

The paper is organized as follows. In Section 1 we recall the definitions and some basic properties for translation invariant Banach function spaces (BF-spaces) and Fourier Banach function spaces. In Section 2 we prove that every temperate distribution $f$ can be associated with a function $F$ analytic in a convex tube satisfying (0.1) and (0.2). Analogous results are given in Sections 3 , 5 for the weighted case, inf types and modulation space types, respectively. We use this result in Section 6] to show some further properties of these wave-front sets. In particular we show a result about the relation between wave-front sets of Fourier Banach function types and analytic wave-front sets. 


\section{Preliminaries}

In this section we recall some notations and basic results. The proofs are in general omitted. In what follows we let $\Gamma$ denote an open cone in $\mathbf{R}^{d} \backslash 0$. If $\xi \in \mathbf{R}^{d} \backslash 0$ is fixed, then an open cone which contains $\xi$ is sometimes denoted by $\Gamma_{\xi}$.

Assume that $\omega, v \in L_{l o c}^{\infty}\left(\mathbf{R}^{d}\right)$ are positive functions. Then $\omega$ is called $v$-moderate if

$$
\omega(x+y) \leq C \omega(x) v(y)
$$

for some constant $C$ which is independent of $x, y \in \mathbf{R}^{d}$. If $v$ in (1.1) can be chosen as a polynomial, then $\omega$ is called polynomially moderate. We let $\mathscr{P}\left(\mathbf{R}^{d}\right)$ be the set of all polynomially moderated functions on $\mathbf{R}^{d}$. We say that $v$ is submultiplicative when (1.1) holds with $\omega=v$. Throughout we assume that the submultiplicative weights are even. If $\omega(x, \xi) \in \mathscr{P}\left(\mathbf{R}^{2 d}\right)$ is constant with respect to the $x$-variable $(\xi$ variable), then we sometimes write $\omega(\xi)(\omega(x))$ instead of $\omega(x, \xi)$. In this case we consider $\omega$ as an element in $\mathscr{P}\left(\mathbf{R}^{2 d}\right)$ or in $\mathscr{P}\left(\mathbf{R}^{d}\right)$ depending on the situation.

For any weight $\omega$ in $\mathscr{P}\left(\mathbf{R}^{d}\right)$ we let $L_{(\omega)}^{p}\left(\mathbf{R}^{d}\right)$ be the set of all $f \in$ $L_{\text {loc }}^{1}\left(\mathbf{R}^{d}\right)$ such that $f \cdot \omega \in L^{p}\left(\mathbf{R}^{d}\right)$.

The Fourier transform $\mathscr{F}$ is the linear and continuous mapping on $\mathscr{S}^{\prime}\left(\mathbf{R}^{d}\right)$ which takes the form

$$
(\mathscr{F} f)(\xi)=\widehat{f}(\xi) \equiv(2 \pi)^{-d / 2} \int_{\mathbf{R}^{d}} f(x) e^{-i\langle x, \xi\rangle} d x
$$

when $f \in L^{1}\left(\mathbf{R}^{d}\right)$. We recall that $\mathscr{F}$ is a homeomorphism on $\mathscr{S}^{\prime}\left(\mathbf{R}^{d}\right)$ which restricts to a homeomorphism on $\mathscr{S}\left(\mathbf{R}^{d}\right)$ and to a unitary operator on $L^{2}\left(\mathbf{R}^{d}\right)$.

Next we recall the definition of Banach function spaces.

Definition 1.1. Assume that $\mathscr{B}$ is a Banach space of complex-valued measurable functions on $\mathbf{R}^{d}$ and that $v \in \mathscr{P}\left(\mathbf{R}^{d}\right)$ is submultiplicative. Then $\mathscr{B}$ is called a (translation) invariant BF-space on $\mathbf{R}^{d}$ (with respect to $v$ ), if there is a constant $C$ such that the following conditions are fulfilled:

(1) $\mathscr{S}\left(\mathbf{R}^{d}\right) \subseteq \mathscr{B} \subseteq \mathscr{S}^{\prime}\left(\mathbf{R}^{d}\right)$ (continuous embeddings);

(2) if $x \in \mathbf{R}^{d}$ and $f \in \mathscr{B}$, then $f(\cdot-x) \in \mathscr{B}$, and

$$
\|f(\cdot-x)\|_{\mathscr{B}} \leq C v(x)\|f\|_{\mathscr{B}} ;
$$

(3) if $f, g \in L_{l o c}^{1}\left(\mathbf{R}^{d}\right)$ satisfy $g \in \mathscr{B}$ and $|f| \leq|g|$ almost everywhere, then $f \in \mathscr{B}$ and

$$
\|f\|_{\mathscr{B}} \leq C\|g\|_{\mathscr{B}}
$$


Assume that $\mathscr{B}$ is a translation invariant BF-space. If $f \in \mathscr{B}$ and $h \in L^{\infty}$, then it follows from (3) in Definition 1.1 that $f \cdot h \in \mathscr{B}$ and

$$
\|f \cdot h\|_{\mathscr{B}} \leq C\|f\|_{\mathscr{B}}\|h\|_{L^{\infty}} .
$$

Remark 1.2. Assume that $\omega_{0}, v, v_{0} \in \mathscr{P}\left(\mathbf{R}^{d}\right)$ are such $v$ and $v_{0}$ are submultiplicative, $\omega_{0}$ is $v_{0}$-moderate, and assume that $\mathscr{B}$ is a translationinvariant BF-space on $\mathbf{R}^{d}$ with respect to $v$. Also let $\mathscr{B}_{0}$ be the Banach space which consists of all $f \in L_{l o c}^{1}\left(\mathbf{R}^{d}\right)$ such that $\|f\|_{\mathscr{B}_{0}} \equiv\left\|f \omega_{0}\right\|_{\mathscr{B}}$ is finite. Then $\mathscr{B}_{0}$ is a translation invariant BF-space with respect to $v_{0} v$.

For future references we note that if $\mathscr{B}$ is a translation invariant BFspace with respect to the submultiplicative weight $v$ on $\mathbf{R}^{d}$, then the convolution map $*$ on $\mathscr{S}\left(\mathbf{R}^{d}\right)$ extends to a continuous mapping from $\mathscr{B} \times L_{(v)}^{1}\left(\mathbf{R}^{d}\right)$ to $\mathscr{B}$, and for some constant $C$ it holds

$$
\|\varphi * f\|_{\mathscr{B}} \leq C\|\varphi\|_{L_{(v)}^{1}}\|f\|_{\mathscr{B}}
$$

when $\varphi \in L_{(v)}^{1}\left(\mathbf{R}^{d}\right)$ and $f \in \mathscr{B}$. In fact, if $f, g \in \mathscr{S}$, then $f * g \in \mathscr{S} \subseteq \mathscr{B}$ in view of the definitions, and Minkowski's inequality gives

$$
\begin{aligned}
& \|f * g\|_{\mathscr{B}}=\left\|\int f(\cdot-y) g(y) d y\right\|_{\mathscr{B}} \\
& \leq \int\|f(\cdot-y)\|_{\mathscr{B}}|g(y)| d y \leq C \int\|f\|_{\mathscr{B}}|g(y) v(y)| d y=C\|f\|_{\mathscr{B}}\|g\|_{L_{(v)}^{1}} .
\end{aligned}
$$

Since $\mathscr{S}$ is dense in $L_{(v)}^{1}$, it follows that $\varphi * f \in \mathscr{B}$ when $\varphi \in L_{(v)}^{1}$ and $f \in \mathscr{S}$, and that (1.2) holds in this case. The result is now a consequence of Hahn-Banach's theorem.

From now on we assume that each translation invariant BF-space $\mathscr{B}$ is such that the convolution map $*$ on $\mathscr{S}\left(\mathbf{R}^{d}\right)$ is uniquely extendable to a continuous mapping from $\mathscr{B} \times L_{(v)}^{1}\left(\mathbf{R}^{d}\right)$ to $\mathscr{B}$, and that $(1.2)$ holds when $\varphi \in L_{(v)}^{1}\left(\mathbf{R}^{d}\right)$ and $f \in \mathscr{B}$. We note that $\mathscr{B}$ can be any mixed and weighted Lebesgue space.

In particular we then have that

$$
\left\|\int_{|y|=1} g(\cdot-y) d y\right\|_{\mathscr{F} \mathscr{B}} \leq C \int_{|y|=1}\|g(\cdot-y)\|_{\mathscr{F} \mathscr{B}} d y .
$$

Assume that $\mathscr{B}$ is a translation invariant BF-space on $\mathbf{R}^{d}$ and $\omega \in$ $\mathscr{P}\left(\mathbf{R}^{d}\right)$. Then we let $\mathscr{F} \mathscr{B}(\omega)$ be the set of all $f \in \mathscr{S}^{\prime}\left(\mathbf{R}^{d}\right)$ such that $\xi \mapsto \widehat{f}(\xi) \omega(\xi)$ belongs to $\mathscr{B}$. It follows that $\mathscr{F} \mathscr{B}(\omega)$ is a Banach space under the norm

$$
\|f\|_{\mathscr{F} \mathscr{B}(\omega)} \equiv\|\widehat{f} \omega\|_{\mathscr{B}} .
$$

Recall that a topological vector space $V \subseteq \mathscr{D}^{\prime}(X)$ is called local if $V \subseteq V_{l o c}$. Here $X \subseteq \mathbf{R}^{d}$ is open, and $V_{l o c}$ consists of all $f \in \mathscr{D}^{\prime}(X)$ such that $\varphi f \in V$ for every $\varphi \in C_{0}^{\infty}(X)$. For future references we note that 
if $\mathscr{B}$ is a translation invariant BF-space on $\mathbf{R}^{d}$, then it follows from $(1.2)$ that $\mathscr{F} \mathscr{B}$ is a local space, i. e.

$$
\mathscr{F} \mathscr{B} \subseteq \mathscr{F} \mathscr{B}_{l o c} \equiv(\mathscr{F} \mathscr{B})_{l o c} .
$$

Let

$$
I(\xi)=\int_{|\omega|=1} e^{-\langle\omega, \xi\rangle} d \omega \quad \text { and } \quad K(z)=(2 \pi)^{-d} \int e^{i\langle z, \xi\rangle} / I(\xi) d \xi .
$$

These functions will play an important role when proving the main results. We therefore explicitly give properties of these functions. These results can be found in Section 8.4 in Hörmander [12].

Let $I$ be given by (1.3) then we have that $I(\xi)=2 \cosh \xi$ for $d=1$ and $I(\xi)=I_{0}\left(\langle\xi, \xi\rangle^{1 / 2}\right)$ for $d>1$. Here

$$
I_{0}(\rho)=c_{d-1} \int_{-1}^{1}\left(1-t^{2}\right)^{(d-3) / 2} e^{t \rho} d t
$$

where $c_{d-1}$ is the area of $S^{d-2}$. Then $I_{0}$ is an even analytic function in $\mathrm{C}$ such that for every $\varepsilon>0$

$$
I_{0}(\rho)=(2 \pi)^{(d-1) / 2} e^{\rho} \rho^{-(d-1) / 2}(1+O(1 / \rho))
$$

if $\rho \rightarrow \infty,|\arg \rho|<\pi / 2-\varepsilon$. Furthermore there is a constant $C$ such that for all $\rho \in \mathbf{C}$ we have that

$$
\left|I_{0}(\rho)\right| \leq C(1+|\rho|)^{-(d-1) / 2} e^{|\operatorname{Re} \rho|} .
$$

The following lemma can be found with proof in [12.

Lemma 1.3. $K(z)$ is an analytic function in the connected open set

$$
\widetilde{\Omega}=\left\{z \in \mathbf{C}^{d} ;\langle z, z\rangle \notin(-\infty,-1]\right\} \supset \Omega .
$$

Here $\Omega=\{z \in \mathbf{C} ;|\operatorname{Im} z|<1\}$. Furthermore, for any closed open cone $\Gamma \subset \widetilde{\Omega}$ such that $\langle z, z\rangle$ is never $\leq 0$ when $z \in \Gamma \backslash 0$ there is some $c>0$ such that $K(z)=O\left(e^{-c|z|}\right)$ when $z \rightarrow \infty$ in $\Gamma$. We have for real $x$ and $y$ that

$$
\begin{aligned}
& |K(x+i y)| \leq K(i y)=(d-1) !(2 \pi)^{-d}(1-|y|)^{-d}(1+O(1-|y|)), \\
& |y| \rightarrow 1^{-} .
\end{aligned}
$$

Furthermore

$$
\left|D^{\beta} K(x+i y)\right| \leq C_{\beta}(1-|y|)^{-n-|\beta|} e^{-c|x|}, \quad|y|<1
$$

holds by Cauchy's inequalities. (Cf. [12].) 


\section{Analytic functions associated With temperate DISTRIBUTIONS}

In this section we show that (0.2) holds. Assume that $\omega \in \mathscr{P}\left(\mathbf{R}^{d}\right)$. We recall that the wave-front sets of weighted Fourier Banach function types $\mathrm{WF}_{\mathscr{F} \mathscr{B}(\omega)}(f)$ consists of all pairs $\left(x_{0}, \xi_{0}\right) \in \mathbf{R}^{d} \times \mathbf{R}^{d} \backslash 0$ such that

$$
|\varphi f|_{\mathscr{F} \mathscr{B}\left(\omega, \Gamma_{\xi_{0}}\right)} \equiv\left\|\mathscr{F}(\varphi f) \chi_{\Gamma_{\xi_{0}}} \omega\right\|_{\mathscr{B}}=\infty
$$

for every open conical neighbourhood $\Gamma_{\xi_{0}}$ of $\xi_{0}$, and $\varphi \in C_{0}^{\infty}$ with $\varphi=1$ in some open neighbourhood $X$ of $x_{0}$. Here $\chi_{\Gamma_{\xi_{0}}}$ is the characteristic function of $\Gamma_{\xi_{0}}$. (Cf. Coriasco, Johansson and Toft [1].)

Let $\mathrm{WF}_{\mathscr{F} \mathscr{B}(\omega)}(f)=\mathrm{WF}_{\mathscr{F} \mathscr{B}}(f)$ if $\omega \equiv 1$.

Definition 2.1. Assume that $f \in \mathscr{D}^{\prime}\left(\mathbf{R}^{d}\right), \mathscr{B}$ is a translation invariant BF-space and $\omega \in \mathscr{P}\left(\mathbf{R}^{d}\right)$. Then $f \in \mathscr{F} \mathscr{B}(\omega)$ at $x_{0}$ if and only if there exists $\varphi \in C_{0}^{\infty}$ with $\varphi \equiv 1$ in a neighbourhood of $x$ such that $\varphi f \in \mathscr{F} \mathscr{B}(\omega)$.

Remark 2.2. For convenience we say that $f \in \mathscr{F} \mathscr{B}$ at $x_{0}$ if the statement in Definition 2.1 is true for $\omega \equiv 1$.

We note that if $f$ belongs to $\mathscr{F} \mathscr{B}(\omega)$ at $x_{0}$ then $\left(x_{0}, \xi_{0}\right) \notin \mathrm{WF}_{\mathscr{F} \mathscr{B}(\omega)}(f)$ for any $\xi_{0} \in \mathbf{R}^{d} \backslash 0$.

Definition 2.3. For $f \in \mathscr{D}^{\prime}(X)$ the singular support sing $\operatorname{supp}_{\mathscr{B}(\omega)} f$ is the smallest closed subset of $X$ such that $f$ is in $\mathscr{F} \mathscr{B}(\omega)$ in the complement.

We use the notation sing $\operatorname{supp}_{\mathscr{B}(\omega)} f=\operatorname{sing} \operatorname{supp}_{\mathscr{B}} f$ when $\omega \equiv 1$.

Theorem 2.4. Assume that $f \in \mathscr{D}^{\prime}\left(\mathbf{R}^{d}\right), \mathscr{B}$ is a translation invariant $B F$-space and $\omega \in \mathscr{P}\left(\mathbf{R}^{d}\right)$. The projection of $\mathrm{WF}_{\mathscr{F} \mathscr{B}(\omega)}(f)$ in $X$ is equal to sing $\operatorname{supp}_{\mathscr{B}(\omega)} f$.

Proof. (a) Assume that $x_{0} \notin \operatorname{sing} \operatorname{supp}_{\mathscr{B}(\omega)}(f)$. Then $f$ belongs to $\mathscr{F} \mathscr{B}(\omega)$ at $x_{0}$. This implies that $\left(x_{0}, \xi_{0}\right) \notin \mathrm{WF}_{\mathscr{F} \mathscr{B}(\omega)}(f)$, for any $\xi_{0} \in$ $\mathbf{R}^{d} \backslash 0$.

(b) Assume that $\left(x_{0}, \xi_{0}\right) \notin \mathrm{WF}_{\mathscr{F} \mathscr{B}(\omega)}(f)$ for all $\xi_{0} \in \mathbf{R}^{d} \backslash 0$. Then by the compactness of unit sphere we can choose a neighbourhood $K$ of $x_{0}$ such that $\mathrm{WF}_{\mathscr{F} \mathscr{B}(\omega)}(f) \cap\left(K \times \mathbf{R}^{d}\right)=\emptyset$. This implies that we can choose a function $\varphi_{x_{0}} \in C_{0}^{\infty}$ which is equal to 1 in a neighbourhood $X$ of $x_{0}$ such that $\varphi_{x_{0}} f \in \mathscr{F} \mathscr{B}(\omega)$. Hence $x_{0} \notin \operatorname{sing} \operatorname{supp}_{\mathscr{B}(\omega)}(f)$.

The next theorem is given without proof since the result follows directly from Theorem 8.4.8 in Hörmander [12] together with the observation that $\mathrm{WF}_{\mathscr{F} \mathscr{B}}(f) \subseteq \mathrm{WF}(f)$.

Theorem 2.5. Let $X \subseteq \mathbf{R}^{d}$ be open, $\Gamma$ an open convex cone in $\mathbf{R}^{d}$ and let

$$
Z=\left\{z \in \mathbf{C}^{d} ; \operatorname{Re} z \in \underset{6}{X} \operatorname{Im} z \in \Gamma,|\operatorname{Im} z|<\gamma\right\}
$$


for some $\gamma>0$. Also let $F$ be an analytic function in $Z$ such that

$$
|F(z)| \leq C|\operatorname{Im} z|^{-N}, z \in Z .
$$

Then $F(\cdot+i y)$ has the limit $F_{0} \in \mathscr{D}^{\prime N+1}(X)$ as $y \in \Gamma$ tends to zero and $\mathrm{WF}_{\mathscr{F} \mathscr{B}}\left(F_{0}\right) \subset X \times\left(\Gamma^{\circ} \backslash 0\right)$, where $\Gamma^{\circ}$ is the dual cone of $\Gamma$. Furthermore $F=0$ if $F_{0}=0$.

Next we associate the temperate distribution $f$ with a function $F$ analytic in the convex cone $\Omega=\left\{z \in \mathbf{C}^{d} ;|\operatorname{Im} z|<1\right\}$ such that

$$
f=\int_{|\xi|=1} F(\cdot+i \xi) d \xi
$$

We recall the following result from Hörmander [12, Theorem 8.4.11].

Theorem 2.6. Let $K$ be given by (1.3) . If $f \in \mathscr{S}^{\prime}\left(\mathbf{R}^{d}\right)$ and $F=K * f$, then $F$ is analytic in $\Omega=\{z ;|\operatorname{Im} z|<1\}$ and for some $C, a, b$

$$
|F(z)| \leq C(1+|z|)^{a}(1-|\operatorname{Im} z|)^{-b}, z \in \Omega .
$$

The boundary values $F(\cdot+i \xi)$ are continuous functions of $\xi \in S^{d-1}$ with values in $\mathscr{S}^{\prime}\left(\mathbf{R}^{d}\right)$, and

$$
\langle f, \phi\rangle=\int\langle F(\cdot+i \xi), \phi\rangle d \xi, \quad \phi \in \mathscr{S} .
$$

Conversely, if F satisfies (2.1), then (2.2) defines a distribution $f \in \mathscr{S}^{\prime}$ with $F=K * f$.

Next we give the main theorem.

Theorem 2.7. Assume that $f$ and $F$ satisfies the conditions in Theorem 2.6. Then we have that

$\left(\mathbf{R}^{d} \times S^{d-1}\right) \cap \mathrm{WF}_{\mathscr{F} \mathscr{B}}(f)=\{(x, \xi) ;|\xi|=1, F$ is not in $\mathscr{F} \mathscr{B}$ at $x-i \xi\}$.

We remark that $F$ is in $\mathscr{F} \mathscr{B}$ at $x-i \xi$ if for some neighbourhood $V$ of $(x, \xi)$ there exists some localization $\varphi \in C_{0}^{\infty}$ with $\varphi=1$ in $V$ such that $\varphi f \in \mathscr{F} \mathscr{B}$. Before the proof we note that $C^{L}$ is a subset of $\mathscr{F} \mathscr{B}$.

Proof. First assume that $\left(x_{0}, \xi_{0}\right) \notin \mathrm{WF}_{\mathscr{F} \mathscr{B}}(f)$ and $\left|\xi_{0}\right|=1$. Then we want to show that $F=K * f \in \mathscr{F} \mathscr{B}$ at $x_{0}-i \xi_{0}$. By the hypothesis there exist $r>0$ and $\varphi_{x_{0}} \in C_{0}^{\infty}$ such that $\varphi_{x_{0}}(x)=1$ if $\left|x-x_{0}\right|<r$ and an open conical neighbourhood $\Gamma_{\xi_{0}}$ of $\xi_{0}$ such that

$$
\left\|\mathscr{F}\left(\varphi_{x_{0}} f\right) \chi_{\Gamma_{\xi_{0}}}\right\|_{\mathscr{B}}<\infty \text {. }
$$

We also recall that since $\varphi_{x_{0}} f$ has compact supports it holds

$$
\left|\mathscr{F}\left(\varphi_{x_{0}} f\right)(\xi)\right| \leq C(1+|\xi|)^{M}, \quad \xi \in \mathbf{R}^{d},
$$

for some fixed constants $C, M \geq 0$. Set $f=\varphi_{x_{0}} f+v$ where $v=$ $f\left(1-\varphi_{x_{0}}\right)$. Then $F=K * f=K *\left(\varphi_{x_{0}} f\right)+K * v$ and

$$
K * v(z)=\underset{7}{\langle K}(z-\cdot), v\rangle .
$$


Now $K(x+i y-t)$ is well-defined when $|y|^{2}<1+|x-t|^{2}$, so it is welldefined and rapidly decreasing with all derivatives when $\left|t-x_{0}\right| \geq r$ if

$$
|y|^{2}<1+\left(r-\left|x-x_{0}\right|\right)^{2}, \quad\left|x-x_{0}\right|<r .
$$

(Cf. Lemma 8.4.10 and Theorem 8.4.11 in [12].) It follows that $K * v$ is analytic and bounded in compact subsets of the set defined by (2.3), which is a neighbourhood of $x_{0}-i \xi_{0}$. Then it follows that $K * v$ belongs to $\mathscr{F} \mathscr{B}$ at $x_{0}-i \xi_{0}$.

Next we consider $K *\left(\varphi_{x_{0}} f\right)$. It is left to prove that $K *\left(\varphi_{x_{0}} f\right)$ belongs to $\mathscr{F} \mathscr{B}$ at $x_{0}-i \xi_{0}$. The Fourier transform of $K *\left(\varphi_{x_{0}} f\right)(\cdot+i y)$

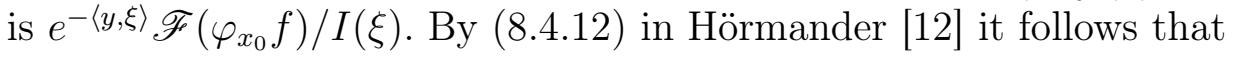

$$
\frac{1}{|I(\xi)|} \leq C e^{-|\xi|}(1+|\xi|)^{(d-1) / 2}
$$

Using this we conclude that

$$
\begin{gathered}
\left\|K *\left(\varphi_{x_{0}} f\right)\right\|_{\mathscr{F} \mathscr{B}}=\left\|e^{-\langle y, \cdot} \mathscr{F}\left(\varphi_{x_{0}} f\right) / I(\cdot)\right\|_{\mathscr{B}} \\
\leq C_{1}\left\|e^{-\langle y, \cdot\rangle-|\cdot|}(1+|\cdot|)^{(d-1) / 2} \mathscr{F}\left(\varphi_{x_{0}} f\right)\right\|_{\mathscr{B}} \\
\leq C_{2}\left(\left\|e^{-\langle y, \cdot\rangle-|\cdot|}(1+|\cdot|)^{(d-1) / 2} \mathscr{F}\left(\varphi_{x_{0}} f\right) \chi_{\Gamma_{\xi_{0}}}\right\|_{\mathscr{B}}+\right. \\
\left.\left\|e^{-\langle y, \cdot\rangle-|\cdot|}(1+|\cdot|)^{(d-1) / 2} \mathscr{F}\left(\varphi_{x_{0}} f\right)\left(1-\chi_{\Gamma_{\xi_{0}}}\right)\right\|_{\mathscr{B}}\right)
\end{gathered}
$$

For the first part in the right-hand side of (2.4) we recognize that for every $y$ such that $|y|<1 \sup _{\xi} e^{-\langle y, \xi\rangle-|\xi|}(1+|\xi|)^{(d-1) / 2}<\infty$ and therefore

$$
\left\|e^{-\langle y \cdot \cdot\rangle-|\cdot|}(1+|\cdot|)^{(d-1) / 2} \mathscr{F}\left(\varphi_{x_{0}} f\right) \chi_{\Gamma_{\xi_{0}}}\right\|_{\mathscr{B}}<\infty .
$$

Then for the second part we have that

$$
\begin{aligned}
\left\|e^{-\langle y, \cdot\rangle-|\cdot|}(1+|\cdot|)^{(d-1) / 2} \mathscr{F}\left(\varphi_{x_{0}} f\right)\left(1-\chi_{\Gamma_{\xi_{0}}}\right)\right\|_{\mathscr{B}} \\
\quad \leq C\left\|e^{-\langle y, \cdot-|\cdot|}(1+|\cdot|)^{M+(d-1) / 2}\left(1-\chi_{\Gamma_{\xi_{0}}}\right)\right\|_{\mathscr{B}} .
\end{aligned}
$$

Choose $\varepsilon>0$ such that $\left\langle\xi_{0}, \xi\right\rangle<(1-2 \varepsilon)|\xi|$ when $\xi \notin \Gamma_{\xi_{0}}$. Then

$$
\langle y, \xi\rangle+|\xi|>\varepsilon|\xi|
$$

if $\xi \notin \Gamma_{\xi_{0}}$ and $\left|y+\xi_{0}\right|<\varepsilon$. Hence we obtain

$$
\begin{aligned}
\| e^{-\langle y, \cdot\rangle-|\cdot|}(1+|\cdot|)^{M+(d-1) / 2}(1 & \left.-\chi_{\Gamma_{\xi_{0}}}\right) \|_{\mathscr{B}} \\
& \leq C\left\|e^{-\varepsilon|\cdot|}(1+|\cdot|)^{M+(d-1) / 2}\right\|_{\mathscr{B}}<\infty
\end{aligned}
$$

This completes the first part of the proof.

For the second part of the proof we need the following lemma. 
Lemma 2.8. Let $d \mu$ be a measure on $S^{d-1}$ and $\Gamma$ an open convex cone such that

$$
\langle y, \xi\rangle<0 \text { when } 0 \neq y \in \bar{\Gamma}, \xi \in \operatorname{supp} d \mu .
$$

If $F$ is analytic in $\Omega$ and satisfies (2.1), then

$$
F_{1}(z)=\int F(z+i \xi) d \mu(\xi)
$$

is analytic and $\left|F_{1}(z)\right| \leq C^{\prime}(1+|\operatorname{Re} z|)^{a}|\operatorname{Im} z|^{-b}$ when $\operatorname{Im} z \in \Gamma$ and $|\operatorname{Im} z|$ is small enough.

For every measure $d \mu$ on $S^{d-1}$ we have

$\mathrm{WF}_{\mathscr{F} \mathscr{B}}\left(F_{\mu}\right) \subset\{(x, \zeta) ;-\zeta /|\zeta| \in \operatorname{supp} d \mu$ and $F \notin \mathscr{F} \mathscr{B}$ at $x-i \zeta /|\zeta|\}$

Here $F_{\mu}=\int F(\cdot+i \xi) d \mu(\xi)$.

Proof. The first statement was proved by Hörmander in [12, Theorem 8.4.12]. Let $\Gamma^{\circ}$ be the dual cone of $\Gamma$. By Theorem 2.5] it follows that

$$
\mathrm{WF}_{\mathscr{F} \mathscr{B}}\left(F_{\mu}\right) \subset \mathbf{R}^{d} \times \Gamma^{\circ} .
$$

Assume that

$$
x_{0} \in\{x ; F \in \mathscr{F} \mathscr{B} \text { at } x+i \xi \text { for every } \xi \in \operatorname{supp} d \mu\} .
$$

Then we have that for every $\xi_{0} \in \operatorname{supp} d \mu$ there exists an open neighbourhood $U_{\xi_{0}}$ of $\left(x_{0}, \xi_{0}\right)$ and a function $\varphi_{\xi_{0}} \in C_{0}^{\infty}$ with $\operatorname{supp} \varphi_{\xi_{0}} \subseteq U_{\xi_{0}}$ such that $x \mapsto \varphi_{\xi_{0}} F \in \mathscr{F} \mathscr{B}$. Since the set

$$
\left\{x_{0}+i \xi ; \xi \in \operatorname{supp} d \mu\right\}
$$

is compact, it follows from arguments about compactness that there exist finitely many points $\xi_{j}$ such that

$$
\left\{x_{0}+i \xi ; \xi \in \operatorname{supp} d \mu\right\} \subseteq \bigcup U_{\xi_{j}} .
$$

For every $\xi_{j}$ we choose an open neighbourhood $X_{\xi_{j}}$ of $x_{0}$ and let $X=$ $\bigcap X_{\xi_{j}}$. Then we can choose $\varphi_{0} \in C_{0}^{\infty}$ equal to one in the neighbourhood $X$ of $x_{0}$ such that $x \mapsto \varphi_{0} F \in \mathscr{F} \mathscr{B}$. Furthermore, we have that there exists $\varphi \in C_{0}^{\infty}$, with support in a neighbourhood of $x_{0}$, such that

$$
\begin{aligned}
&\left\|\varphi F_{\mu}\right\|_{\mathscr{F} \mathscr{B}}=\left\|\int \varphi F(\cdot+i \xi) d \mu\right\|_{\mathscr{F} \mathscr{B}} \\
& \leq \int\|\varphi F(\cdot+i \xi)\|_{\mathscr{F} \mathscr{B}} d \mu<\infty .
\end{aligned}
$$

Then $\varphi F_{\mu} \in \mathscr{F} \mathscr{B}$ at $x_{0}$.

From the arguments above it follows that $\operatorname{sing} \operatorname{supp}_{\mathscr{B}}\left(F_{\mu}\right) \subset\{x ; F$ is not in $\mathscr{F} \mathscr{B}$ at $x+i \xi$ for some $\xi \in \operatorname{supp} d \mu\}$. Then we may write $d \mu=\sum d \mu_{j}$ where $\operatorname{supp} d \mu_{j}$ is contained in the intersection of supp $d \mu$ and a narrow open convex cone $V_{j}$. Applying 
the result just proved with $d \mu$ replaced by $d \mu_{j}$ and $\Gamma$ replaced by the interior of the dual cone $-V_{j}^{\circ}$ we obtain

$\mathrm{WF}_{\mathscr{F} \mathscr{B}}\left(F_{\mu}\right) \subset \bigcup\left\{(x, \zeta) ;-\zeta /|\zeta| \in \bar{V}_{j}, F \notin \mathscr{F} \mathscr{B}\right.$ at $x+i \xi$ for some $\left.\xi \in V_{j}\right\}$.

If $-\zeta /|\zeta| \notin \operatorname{supp} d \mu$ or $F \in \mathscr{F} \mathscr{B}$ at $x-i \zeta /|\zeta|$ we can choose the covering so that $-\zeta /|\zeta| \notin \bar{V}_{j}$ for every $j$ or for all $j \neq 1$ while $F \in \mathscr{F} \mathscr{B}$ at $x+i \xi$ for every $\xi \in V_{1}$. In both cases it follows that $(x, \xi) \notin \mathrm{WF}_{\mathscr{F} \mathscr{B}}\left(F_{\mu}\right)$ which proves (2.5). This completes the proofs of Lemma 2.8 and Theorem 2.7 .

The following Corollary is an analogue to Corollary 8.4.13 in Hörmander [12.

Corollary 2.9. Let $\Gamma_{1}, \ldots, \Gamma_{m}$ be closed cones in $\mathbf{R}^{d} \backslash 0$ such that

$$
\bigcup_{j=1}^{m} \Gamma_{j}=\mathbf{R}^{d} \backslash 0
$$

For every $f \in \mathscr{S}^{\prime}\left(\mathbf{R}^{d}\right)$ there exists a decomposition $f=\sum_{j=1}^{m} f_{j}$, where $f_{j} \in \mathscr{S}^{\prime}$ and

$$
\mathrm{WF}_{\mathscr{F} \mathscr{B}}\left(f_{j}\right) \subseteq \mathrm{WF}_{\mathscr{F} \mathscr{B}}(f) \cap\left(\mathbf{R}^{d} \times \Gamma_{j}\right)
$$

If there exists another decomposition $f=\sum_{j=1}^{m} f_{j}^{\prime}$ which also satisfies the conditions above, then $f_{j}^{\prime}=f_{j}+\sum_{k=1}^{m} f_{j k}$ where $f_{j k} \in \mathscr{S}^{\prime}, f_{j k}=$ $-f_{k j}$ and

$$
\mathrm{WF}_{\mathscr{F} \mathscr{B}}\left(f_{j k}\right) \subset W F_{\mathscr{F} \mathscr{B}}(f) \cap\left(\mathbf{R}^{d} \times\left(\Gamma_{j} \cap \Gamma_{k}\right)\right) .
$$

Proof. Let $\phi_{j}$ be the characteristic function on $\Gamma_{j} \backslash\left(\Gamma_{1} \cup \cdots \cup \Gamma_{j-1}\right)$. Then since $\operatorname{supp} \phi_{j} \cap \operatorname{supp} \phi_{k}=\emptyset$ for every $j \neq k$ and

$$
\bigcup_{j=1}^{m} \Gamma_{j} \backslash\left(\Gamma_{1} \cup \cdots \cup \Gamma_{j-1}\right)=\bigcup_{j=1}^{m} \Gamma_{j}=\mathbf{R}^{d} \backslash 0
$$

it follows that $\sum \phi_{j}=1$ in $\mathbf{R}^{d} \backslash 0$. Let $F=K * f$ and $F_{j}=K *\left(f_{j}^{\prime}-f_{j}\right)$. Then

$$
\sum_{j=1}^{m} F_{j}=\sum_{j=1}^{m}\left(K *\left(f_{j}^{\prime}-f_{j}\right)\right)=K *\left(\sum_{j=1}^{m} f_{j}^{\prime}-\sum_{j=1}^{m} f_{j}\right)=0 .
$$

Let

$$
f_{j}=\int F(\cdot-i \xi) \phi_{j}(\xi) d \xi
$$

and

$$
f_{j k}=\int F_{j}(\cdot-i \xi) \phi_{k}(\xi) d \xi-\int F_{k}(\cdot-i \xi) \phi_{j}(\xi) d \xi
$$


Then it follows by straight-forward calculations that $f_{j}^{\prime}=f_{j}+\sum_{k=1}^{m} f_{j k}$ and $f_{j k}=-f_{k j}$. More precisely we have that

$$
\begin{aligned}
\sum_{k=1}^{m} f_{j k}=\int F_{j}(\cdot-i \xi) \sum_{k=1}^{m} \phi_{k}(\xi) d \xi- & \int \sum_{k=1}^{m} F_{k}(\cdot-i \xi) \phi_{j}(\xi) d \xi \\
& =\int F_{j}(\cdot-i \xi) d \xi=f_{j}^{\prime}-f_{j} .
\end{aligned}
$$

From Theorem 2.7 and Lemma 2.8 it follows that (2.6) holds using that $\phi_{j}$ has support in $\Gamma_{j} \cap S^{d-1}$ and letting $d \mu_{j}(\xi)=\phi_{j}(\xi) d \xi$. Use the measure defined above and treat the integrals on the right-hand side of (2.8) separately. By using the arguments above we see that the wave-front sets of these integrals are contained in

$$
\left(\mathrm{WF}_{\mathscr{F} \mathscr{B}}\left(f_{j}\right) \cup \mathrm{WF}_{\mathscr{F} \mathscr{B}}\left(f_{j}^{\prime}\right)\right) \cap\left(\mathbf{R}^{d} \times \Gamma_{k}\right)
$$

and

$$
\left(\mathrm{WF}_{\mathscr{F} \mathscr{B}}\left(f_{k}\right) \cup \mathrm{WF}_{\mathscr{F} \mathscr{B}}\left(f_{k}^{\prime}\right)\right) \cap\left(\mathbf{R}^{d} \times \Gamma_{j}\right)
$$

respectively. Now (2.7) follows immediately from this together with the fact that $f_{j}$ and $f_{j}^{\prime}$ satisfies (2.6).

\section{WAVE-Front SETS of Weighted Fourier BF-TyPeS}

In this section we consider weighted Fourier BF-spaces and prove results analogous to the non-weighted case. We start by assuming that $\mathscr{B}$ is a translation invariant BF-space and $\omega \in \mathscr{P}\left(\mathbf{R}^{d}\right)$. Then let $\mathscr{B}_{1}=\mathscr{B}(\omega)$. By the following lemma we see that there is no restriction to assume that $\omega$ is $v_{0}$-moderated for some $v_{0} \in \mathscr{P}\left(\mathbf{R}^{d}\right)$ which is submultiplicative.

Lemma 3.1. Assume that $\omega \in \mathscr{P}\left(\mathbf{R}^{d}\right)$. Then there exists $v_{0} \in \mathscr{P}\left(\mathbf{R}^{d}\right)$ such that $v_{0}$ is submultiplicative and

$$
\omega(x) \leq C v_{0}(x),
$$

where the constant $C>0$ is independent of $x \in \mathbf{R}^{d}$.

Proof. Assume that $\omega \in \mathscr{P}\left(\mathbf{R}^{d}\right)$. Then we can choose constants $N$ and $C$ large enough such that

$$
\omega(x) \leq C w(0)\langle x\rangle^{N} .
$$

Note that $C$ and $N$ do not depend on $x \in \mathbf{R}^{d}$. From the fact that

$$
\langle x+y\rangle \leq 2\langle x\rangle\langle y\rangle,
$$

for every $x, y \in \mathbf{R}^{d}$ it follows that $\langle x\rangle^{N}$ is submultiplicative and polynomially moderated. 
Assume that $\omega, v \in \mathscr{P}\left(\mathbf{R}^{d}\right)$ and that $v$ is submultiplicative. By the previous lemma we may choose $v_{0} \in \mathscr{P}\left(\mathbf{R}^{d}\right)$ such that $v_{0}$ is submultiplicative and $\omega$ is $v_{0}$-moderate. Also assume that $\mathscr{B}$ is a translation invariant BF-space on $\mathbf{R}^{d}$ with respect to $v$ and let $\mathscr{B}_{1}$ be the Banach space which consists of all $f \in L_{\mathrm{loc}}^{1}\left(\mathbf{R}^{d}\right)$ such that $\|f\|_{\mathscr{B}_{1}} \equiv\|f \omega\|_{\mathscr{B}}$ is finite. We recall from Remark 1.2 in [1] that $\mathscr{B}_{1}$ then is a translation invariant $\mathrm{BF}$-space with respect to $v_{0} v$.

Next we state the main results in the weighted version. Since these results are obtained directly using the statement above together with the analogous results for the non-weighted case we give the following results without proofs.

Theorem 2.7. Assume that $f$ and $F$ satisfies the conditions in Theorem [2.6. Also assume that $\omega, v, v_{0} \in \mathscr{P}\left(\mathbf{R}^{d}\right)$ are such that $v$ and $v_{0}$ are submultiplicative, $\omega$ is $v_{0}$-moderate, and assume that $\mathscr{B}$ is a translation invariant BF-space on $\mathbf{R}^{d}$ with respect to $v$. Then we have that

$\left(\mathbf{R}^{d} \times S^{d-1}\right) \cap \mathrm{WF}_{\mathscr{F} \mathscr{B}(\omega)}(f)=\{(x, \xi) ;|\xi|=1, F$ is not in $\mathscr{F} \mathscr{B}(\omega)$ at $x-i \xi\}$.

Lemma 2.8. Let $\mathscr{B}$ and $\omega, v$ and $v_{0}$ be defined as in the previous theorem. Also let $d \mu$ be a measure on $S^{d-1}$ and $\Gamma$ an open convex cone such that

$$
\langle y, \xi\rangle<0 \text { when } 0 \neq y \in \bar{\Gamma}, \xi \in \operatorname{supp} d \mu .
$$

If $F$ is analytic in $\Omega$ and satisfies (2.1), then

$$
F_{1}(z)=\int F(z+i \xi) d \mu(\xi)
$$

is analytic and $\left|F_{1}(z)\right| \leq C^{\prime}(1+|\operatorname{Re} z|)^{a}|\operatorname{Im} z|^{-b}$ when $\operatorname{Im} z \in \Gamma$ and $|\operatorname{Im} z|$ is small enough.

For every measure $d \mu$ on $S^{d-1}$ we have

$\mathrm{WF}_{\mathscr{F} \mathscr{B}(\omega)}\left(F_{\mu}\right) \subset\{(x, \zeta) ;-\zeta /|\zeta| \in \operatorname{supp} d \mu$ and $F \notin \mathscr{F} \mathscr{B}(\omega)$ at $x-i \zeta /|\zeta|\}$

Here $F_{\mu}=\int F(\cdot+i \xi) d \mu(\xi)$.

Corollary 2.9. Let $\mathscr{B}$ and $\omega, v$ and $v_{0}$ be defined as in Theorem [2.7'. Also let $\Gamma_{1}, \ldots, \Gamma_{m}$ be closed cones in $\mathbf{R}^{d} \backslash 0$ such that

$$
\bigcup_{j=1}^{m} \Gamma_{j}=\mathbf{R}^{d} \backslash 0 .
$$

For every $f \in \mathscr{S}^{\prime}\left(\mathbf{R}^{d}\right)$ there exists a decomposition $f=\sum_{j=1}^{m} f_{j}$, where $f_{j} \in \mathscr{S}^{\prime}$ and

$$
\mathrm{WF}_{\mathscr{F} \mathscr{B}(\omega)}\left(f_{j}\right) \subseteq \mathrm{WF}_{\mathscr{F} \mathscr{B}(\omega)}(f) \cap\left(\mathbf{R}^{d} \times \Gamma_{j}\right)
$$

If there exists another decomposition $f=\sum_{j=1}^{m} f_{j}^{\prime}$ which also satisfies the conditions above, then $f_{j}^{\prime}=f_{j}+\sum_{k=1}^{m} f_{j k}$ where $f_{j k} \in \mathscr{S}^{\prime}, f_{j k}=$ $-f_{k j}$ and

$$
\mathrm{WF}_{\mathscr{F} \mathscr{B}(\omega)}\left(f_{j k}\right) \subset W F_{\mathscr{F} \mathscr{B}(\omega)}(f) \cap\left(\mathbf{R}^{d} \times\left(\Gamma_{j} \cap \Gamma_{k}\right)\right) .
$$




\section{WAVE-FRONT SETS OF INF TYPE}

In this section we show analogous results for wave-front sets of inf types. We recall the definitions of these types of wave-front sets from Coriasco, Johansson and Toft 1 . Let $\mathscr{B}_{j}$ be a translation invariant BF-space on $\mathbf{R}^{d}$ and $\omega_{j} \in \mathscr{P}\left(\mathbf{R}^{d}\right)$, when $j$ belongs to some index set $J$, and consider the array of spaces, given by

$$
\left(\mathcal{B}_{j}\right) \equiv\left(\mathcal{B}_{j}\right)_{j \in J}, \quad \text { where } \quad \mathcal{B}_{j}=\mathscr{F}_{\mathscr{B}_{j}}\left(\omega_{j}\right), \quad j \in J .
$$

We recall that the wave-front sets of inf types $\mathrm{WF}_{\left(\mathcal{B}_{j}\right)}^{\mathrm{inf}}(f)=\mathrm{WF}_{\left(\mathscr{F} \mathscr{B}_{j}\left(\omega_{j}\right)\right)}^{\inf }(f)$ consists of all pairs $\left(x_{0}, \xi_{0}\right) \in \mathbf{R}^{d} \times \mathbf{R}^{d} \backslash 0$ such that for every open conical neighbourhood $\Gamma_{\xi_{0}}$ of $\xi_{0}$, every $\varphi \in C_{0}^{\infty}$ with $\varphi=1$ in some open neighbourhood $X$ of $x_{0}$ and for every $j \in J$ it holds that

$$
|\varphi f|_{\mathscr{F} \mathscr{B}_{j}\left(\Gamma_{\xi_{0}}\right)} \equiv\left\|\mathscr{F}(\varphi f) \chi_{\Gamma_{\xi_{0}}}\right\|_{\mathscr{B}_{j}\left(\omega_{j}\right)}=\infty .
$$

Here $\chi_{\Gamma_{\xi_{0}}}$ is the characteristic function of $\Gamma_{\xi_{0}}$.

Before stating analogous results to those for wave-front sets of Fourier BF-spaces we compare the wave-front sets of Fourier BF-spaces with the wave-front sets of inf types defined above.

Since $\left(x_{0}, \xi_{0}\right) \in \mathrm{WF}_{\left(\mathcal{B}_{j}\right)}^{\inf }(f)$ if and only if $\left(x_{0}, \xi_{0}\right) \in \mathrm{WF}_{\mathcal{B}_{j}}(f)$ for every $j \in J$, it follows that

$$
\mathrm{WF}_{\left(\mathcal{B}_{j}\right)}^{\inf }(f)=\bigcap_{j} \mathrm{WF}_{\mathcal{B}_{j}}(f)
$$

Theorem 2.7$]^{\prime}$. Let $\mathscr{B}_{j}$ be a translation invariant BF-space on $\mathbf{R}^{d}$ and $\omega \in \mathscr{P}\left(\mathbf{R}^{d}\right)$ for every $j \in J$. Also let $\mathcal{B}_{j}$ be defined as in (4.1) and let $f$ and $F$ satisfy the conditions in Theorem 2.6. Then we have that

$$
\left(\mathbf{R}^{d} \times S^{d-1}\right) \cap \mathrm{WF}_{\left(\mathcal{B}_{j}\right)}^{\inf }(f)=\left\{(x, \xi) ;|\xi|=1, F \text { is not in } \bigcup_{j} \mathcal{B}_{j} \text { at } x-i \xi\right\} .
$$

Proof. We have that

$$
\begin{aligned}
\left(\mathbf{R}^{d} \times S^{d-1}\right) \cap \mathrm{WF}_{\left(\mathcal{B}_{j}\right)}^{\mathrm{inf}}(f)=\bigcap_{j}\left(\left(\mathbf{R}^{d} \times S^{d-1}\right) \cap \mathrm{WF}_{\mathcal{B}_{j}}(f)\right) \\
=\bigcap_{j}\left\{(x, \xi) ;|\xi|=1, F \text { is not in } \mathcal{B}_{j} \text { at } x-i \xi\right\} \\
=\left\{(x, \xi) ;|\xi|=1, F \text { is not in } \bigcup_{j} \mathcal{B}_{j} \text { at } x-i \xi\right\} .
\end{aligned}
$$

The proof is complete

Lemma 4.1. Let $d \mu$ be a measure on $S^{d-1}$ and $\Gamma$ an open convex cone such that

$$
\langle y, \xi\rangle<0 \text { when } 0 \neq y \in \bar{\Gamma}, \xi \in \operatorname{supp} d \mu \text {. }
$$


If $F$ is analytic in $\Omega$ and satisfies (2.1), then

$$
F_{1}(z)=\int F(z+i \xi) d \mu(\xi)
$$

is analytic and $\left|F_{1}(z)\right| \leq C^{\prime}(1+|\operatorname{Re} z|)^{a}|\operatorname{Im} z|^{-b}$ when $\operatorname{Im} z \in \Gamma$ and $|\operatorname{Im} z|$ is small enough.

For every measure $d \mu$ on $S^{d-1}$ we have

$$
\mathrm{WF}_{\left(\mathcal{B}_{j}\right)}^{\inf _{1}}\left(F_{\mu}\right) \subset\left\{(x, \zeta) ;-\zeta /|\zeta| \in \operatorname{supp} d \mu \text { and } F \notin \bigcup_{j} \mathcal{B}_{j} \text { at } x-i \zeta /|\zeta|\right\}
$$

Here $F_{\mu}=\int F(\cdot+i \xi) d \mu(\xi)$.

The following Corollary is an analogue to Corollary 8.4.13 in Hörmander [12].

Corollary 4.2. Let $\Gamma_{1}, \ldots, \Gamma_{m}$ be closed cones in $\mathbf{R}^{d} \backslash 0$ such that

$$
\bigcup_{j=1}^{m} \Gamma_{j}=\mathbf{R}^{d} \backslash 0 .
$$

For every $f \in \mathscr{S}^{\prime}\left(\mathbf{R}^{d}\right)$ there exists a decomposition $f=\sum_{j=1}^{m} f_{j}$, where $f_{j} \in \mathscr{S}^{\prime}$ and

$$
\mathrm{WF}_{\left(\mathcal{B}_{j}\right)}^{\inf }\left(f_{j}\right) \subseteq \mathrm{WF}_{\left(\mathcal{B}_{j}\right)}^{\inf }(f) \cap\left(\mathbf{R}^{d} \times \Gamma_{j}\right) .
$$

If there exists another decomposition $f=\sum_{j=1}^{m} f_{j}^{\prime}$ which also satisfies the conditions above, then $f_{j}^{\prime}=f_{j}+\sum_{k=1}^{m} f_{j k}$ where $f_{j k} \in \mathscr{S}^{\prime}, f_{j k}=$ $-f_{k j}$ and

$$
\mathrm{WF}_{\left(\mathcal{B}_{j}\right)}^{\mathrm{inf}}\left(f_{j k}\right) \subset \mathrm{WF}_{\left(\mathcal{B}_{j}\right)}^{\inf }(f) \cap\left(\mathbf{R}^{d} \times\left(\Gamma_{j} \cap \Gamma_{k}\right)\right) .
$$

\section{WAVE-FRONT SETS OF MODULATION SPACE TYPES}

In this section we show that the results obtained for wave-front sets of Fourier BF-space types also hold for wave-front sets of modulation space types.

We start by defining general types of modulation spaces. Let (the window) $\phi \in \mathscr{S}^{\prime}\left(\mathbf{R}^{d}\right) \backslash 0$ be fixed, and let $f \in \mathscr{S}^{\prime}\left(\mathbf{R}^{d}\right)$. Then the shorttime Fourier transform $V_{\phi} f$ is the element in $\mathscr{S}^{\prime}\left(\mathbf{R}^{2 d}\right)$, defined by the formula

$$
\left(V_{\phi} f\right)(x, \xi) \equiv \mathscr{F}(f \cdot \overline{\phi(\cdot-x)})(\xi) .
$$

We usually assume that $\phi \in \mathscr{S}\left(\mathbf{R}^{d}\right)$, and in this case the short-time Fourier transform $\left(V_{\phi} f\right)$ takes the form

$$
\left(V_{\phi} f\right)(x, \xi)=(2 \pi)^{-d / 2} \int_{\mathbf{R}^{d}} f(y) \overline{\phi(y-x)} e^{-\langle y, \xi\rangle} d y,
$$

when $f \in \mathscr{S}\left(\mathbf{R}^{d}\right)$.

Now let $\mathscr{B}$ be a translation invariant BF-space on $\mathbf{R}^{2 d}$, with respect to $v \in \mathscr{P}\left(\mathbf{R}^{2 d}\right)$. Also let $\phi \in \mathscr{S}\left(\mathbf{R}^{d}\right) \backslash 0$ and $\omega \in \mathscr{P}\left(\mathbf{R}^{2 d}\right)$ be such that 
$\omega$ is $v$-moderate. Then the modulation space $M(\omega)=M(\omega, \mathscr{B})$ is a Banach space with the norm

$$
\|f\|_{M(\omega, \mathscr{B})} \equiv\left\|V_{\phi} f \omega\right\|_{\mathscr{B}}
$$

(cf. [8]).

Assume that $\omega \in \mathscr{P}\left(\mathbf{R}^{2 d}\right)$. We recall that the wave-front sets of modulation space types $\mathrm{WF}_{M(\omega, \mathscr{B})}(f)$ consists of all pairs $\left(x_{0}, \xi_{0}\right) \in$ $\mathbf{R}^{d} \times \mathbf{R}^{d} \backslash 0$ such that

$$
|\varphi f|_{M\left(\omega, \mathscr{B}, \Gamma_{\xi_{0}}\right)} \equiv\left\|V_{\phi}(\varphi f) \chi_{\Gamma_{\xi_{0}}} \omega\right\|_{\mathscr{B}}=\infty
$$

for every open conical neighbourhood $\Gamma_{\xi_{0}}$ of $\xi_{0}$, and $\varphi \in C_{0}^{\infty}$ with $\varphi=1$ in some open neighbourhood $X$ of $x_{0}$. Here $\chi_{\Gamma_{\xi_{0}}}$ is the characteristic function of $\Gamma_{\xi_{0}}$. It can also be showed that wave-front sets of modulation space types and wave-front sets of Fourier BF-types coincide. More precisely, let

$$
\mathscr{B}_{0}=\left\{f \in \mathscr{S}^{\prime}\left(\mathbf{R}^{d}\right): \varphi \otimes f \in \mathscr{B}\right\} .
$$

Then $\mathscr{B}_{0}$ is a translation invariant BF-space on $\mathbf{R}^{d}$, which is independent of the choice of $\varphi$. Furthermore $M(\omega, \mathscr{B})$ and $\mathscr{F} \mathscr{B}_{0}$ are locally the same and

$$
\mathrm{WF}_{\mathscr{F} \mathscr{B}_{0}(\omega)}(f)=\mathrm{WF}_{M(\omega, \mathscr{B})}(f) .
$$

(Cf. Coriasco, Johansson and Toft [1.)

By using the previous results in combination with this we obtain the following results.

Definition 5.1. Assume that $f \in \mathscr{D}^{\prime}\left(\mathbf{R}^{d}\right), \mathscr{B}$ is a translation invariant BF-space and $\omega \in \mathscr{P}\left(\mathbf{R}^{2 d}\right)$. Then $f \in M(\omega, \mathscr{B})$ at $x_{0}$ if and only if there is some neighbourhood $X$ of $x_{0}$ such that for some $\varphi \in C_{0}^{\infty}$ with $\varphi \equiv 1$ in $X$ we have that $\varphi f \in M(\omega, \mathscr{B})$.

We recognize by the arguments before that since the definition above only concerns local properties it holds that $f \in M(\omega, \mathscr{B})$ at $x_{0}$ if and only if $f \in \mathscr{F} \mathscr{B}_{0}(\omega)$ at $x_{0}$, where $\mathscr{B}_{0}$ is given by (5.2).

We note that if $f$ belongs to $M(\omega, \mathscr{B})$ at $x_{0}$ then $\left(x_{0}, \xi_{0}\right) \notin \mathrm{WF}_{M(\omega, \mathscr{B})}(f)$ for any $\xi_{0} \in \mathbf{R}^{d} \backslash 0$.

Definition 5.2. For $f \in \mathscr{D}^{\prime}(X)$ the singular support $\operatorname{sing} \operatorname{supp}_{M(\omega, \mathscr{B})} f$ is the smallest closed subset of $X$ such that $f$ is in $M(\omega, \mathscr{B})$ in the complement.

Theorem 5.3. Assume that $f \in \mathscr{D}^{\prime}\left(\mathbf{R}^{d}\right), \mathscr{B}$ is a translation invariant $B F$-space and $\omega \in \mathscr{P}\left(\mathbf{R}^{2 d}\right)$. The projection of $\mathrm{WF}_{M(\omega, \mathscr{B})}(f)$ in $X$ is equal to sing $\operatorname{supp}_{M(\omega, \mathscr{B})} f$.

Proof. (a) Assume that $x_{0} \notin \operatorname{sing} \operatorname{supp}_{M(\omega, \mathscr{B})}(f)$. Then $f$ belongs to $M(\omega, \mathscr{B})$ at $x_{0}$. This implies that $\left(x_{0}, \xi_{0}\right) \notin \mathrm{WF}_{M(\omega, \mathscr{B})}(f)$, for any $\xi_{0} \in$ $\mathbf{R}^{d} \backslash 0$.

(b) Assume that $\left(x_{0}, \xi_{0}\right) \notin \mathrm{WF}_{M(\omega, \mathscr{B})}(f)$ for all $\xi_{0} \in \mathbf{R}^{d} \backslash 0$. Then we can choose a neighbourhood $K$ of $x_{0}$ such that $\mathrm{WF}_{M(\omega, \mathscr{B})}(f) \cap(K \times$ 
$\left.\mathbf{R}^{d}\right)=\emptyset$. This implies that we can choose a function $\varphi_{x_{0}} \in C_{0}^{\infty}$ which is equal to 1 in a neighbourhood $X$ of $x_{0}$ such that $\varphi_{x_{0}} f \in M(\omega, \mathscr{B})$. Hence $x_{0} \notin \operatorname{sing} \operatorname{supp}_{M(\omega, \mathscr{B})}(f)$.

Next theorem is analogous to Theorem 2.7.

Theorem 2.7. Assume that $f$ and $F$ satisfy the conditions in Theorem 2.6. Also let $\mathscr{B}$ be a translation invariant BF-space and $\omega \in \mathscr{P}\left(\mathbf{R}^{2 d}\right)$. Then we have that

$\left(\mathbf{R}^{d} \times S^{d-1}\right) \cap \mathrm{WF}_{M(\omega, \mathscr{B})}(f)=\{(x, \xi) ;|\xi|=1, F$ is not in $M(\omega, \mathscr{B})$ at $x-i \xi\}$.

We remark that $F$ is in $M(\omega, \mathscr{B})$ at $x-i \xi$ if for some neighbourhood $V$ of $(x, \xi)$ there exists some localization $\varphi \in C_{0}^{\infty}$ with $\varphi=1$ in $V$ such that $\varphi f \in M(\omega, \mathscr{B})$.

Proof. Let $\mathscr{B}_{0}$ be defined as before. Then it follows that

$$
\left(\mathbf{R}^{d} \times S^{d-1}\right) \cap \mathrm{WF}_{M(\omega, \mathscr{B})}(f)=\left(\mathbf{R}^{d} \times S^{d-1}\right) \cap \mathrm{WF}_{\mathscr{F} \mathscr{B}}(\omega)(f) .
$$

From the result in the previous section we also have that

$$
\left(\mathbf{R}^{d} \times S^{d-1}\right) \cap \mathrm{WF}_{\mathscr{F} \mathscr{B}_{0}(\omega)}(f)=\left\{(x, \xi) ;|\xi|=1, F \text { is not in } \mathscr{F}_{\mathscr{B}_{0}}(\omega) \text { at } x-i \xi\right\} .
$$

Now since the right-hand side only concern local properties and $\mathscr{F} \mathscr{B} 0(\omega)$ and $M(\omega, \mathscr{B})$ are locally the same it follows that

$$
\begin{aligned}
& \left\{(x, \xi) ;|\xi|=1, F \text { is not in } \mathscr{F} \mathscr{B}_{0}(\omega) \text { at } x-i \xi\right\} \\
& \quad=\{(x, \xi) ;|\xi|=1, F \text { is not in } M(\omega, \mathscr{B}) \text { at } x-i \xi\}
\end{aligned}
$$

This completes the proof.

By arguments given before it is obvious that Lemma 2.8 and Corollary 2.9 hold also for modulation spaces instead of Fourier BF-spaces. We therefore state the following results without proofs.

Lemma 2.8. Let $\mathscr{B}$ be a translation invariant BF-space and $\omega \in$ $\mathscr{P}\left(\mathbf{R}^{2 d}\right)$. Also let $d \mu$ be a measure on $S^{d-1}$ and $\Gamma$ an open convex cone such that

$$
\langle y, \xi\rangle<0 \text { when } 0 \neq y \in \bar{\Gamma}, \xi \in \operatorname{supp} d \mu .
$$

If $F$ is analytic in $\Omega$ and satisfies (2.1), then

$$
F_{1}(z)=\int F(z+i \xi) d \mu(\xi)
$$

is analytic and $\left|F_{1}(z)\right| \leq C^{\prime}(1+|\operatorname{Re} z|)^{a}|\operatorname{Im} z|^{-b}$ when $\operatorname{Im} z \in \Gamma$ and $|\operatorname{Im} z|$ is small enough.

For every measure $d \mu$ on $S^{d-1}$ we have

$\mathrm{WF}_{M(\omega, \mathscr{B})}\left(F_{\mu}\right) \subset\{(x, \zeta) ;-\zeta /|\zeta| \in \operatorname{supp} d \mu$ and $F \notin M(\omega, \mathscr{B})$ at $x-i \zeta /|\zeta|\}$

Here $F_{\mu}=\int F(\cdot+i \xi) d \mu(\xi)$. 
Corollary 2.9. Let $\mathscr{B}$ be a translation invariant BF-space and $\omega \in$ $\mathscr{P}\left(\mathbf{R}^{2 d}\right)$. Also let $\Gamma_{1}, \ldots, \Gamma_{m}$ be closed cones in $\mathbf{R}^{d} \backslash 0$ such that

$$
\bigcup_{j=1}^{m} \Gamma_{j}=\mathbf{R}^{d} \backslash 0
$$

For every $f \in \mathscr{S}^{\prime}\left(\mathbf{R}^{d}\right)$ there exists a decomposition $f=\sum_{j=1}^{m} f_{j}$, where $f_{j} \in \mathscr{S}^{\prime}$ and

$$
\mathrm{WF}_{M(\omega, \mathscr{B})}\left(f_{j}\right) \subseteq \mathrm{WF}_{M(\omega, \mathscr{B})}(f) \cap\left(\mathbf{R}^{d} \times \Gamma_{j}\right) .
$$

If there exists another decomposition $f=\sum_{j=1}^{m} f_{j}^{\prime}$ which also satisfies the conditions above, then $f_{j}^{\prime}=f_{j}+\sum_{k=1}^{m} f_{j k}$ where $f_{j k} \in \mathscr{S}^{\prime}, f_{j k}=$ $-f_{k j}$ and

$$
\mathrm{WF}_{M(\omega, \mathscr{B})}\left(f_{j k}\right) \subset W F_{M(\omega, \mathscr{B})}(f) \cap\left(\mathbf{R}^{d} \times\left(\Gamma_{j} \cap \Gamma_{k}\right)\right) .
$$

\section{Some AdDitional Properties}

In this section we prove some further properties for the wave-front sets of Fourier Banach types using results from the previous section.

Theorem 6.1. Let $f \in \mathscr{D}^{\prime}(X), X \subseteq \mathbf{R}^{d}$, and $\mathrm{WF}_{\mathscr{F} \mathscr{B}}(f) \subseteq X \times \Gamma^{\circ}$, where $\Gamma^{\circ}$ is the dual of an open convex cone $\Gamma$. If $\overline{X_{1}} \subseteq X$ and $\Gamma_{1}$ is an open convex with $\overline{\Gamma_{1}} \subseteq \Gamma \cup\{0\}$, then there exists a function $F$ that is analytic in $\left\{x+i y ; x \in X_{1}, y \in \Gamma_{1},|y|<\gamma\right\}$, such that

$$
|F(x+i y)|<C|y|^{-N}, \quad y \in \Gamma_{1}, \quad x \in X_{1},
$$

and such that the limit of $F(\cdot-i y)$ in $\Gamma_{1}$, when $y \rightarrow 0$, differs from $f$ by an element in $\mathscr{F} \mathscr{B}\left(X_{1}\right)$.

Proof. Set $v=\chi f$ where $\chi \in C_{0}^{\infty}$ is equal to 1 in $X_{1}$. If $V=K * v$ is defined as in Theorem 2.7, then

$$
\mathrm{WF}_{\mathscr{F} \mathscr{B}}(v)=\mathrm{WF}_{\mathscr{F} \mathscr{B}}(\chi f) \subseteq \mathrm{WF}_{\mathscr{F} \mathscr{B}}(f) \subseteq X \times \Gamma^{\circ}
$$

gives

$$
\complement\left(X \times \Gamma^{\circ}\right) \subseteq \complement \mathrm{WF}_{\mathscr{F} \mathscr{B}}(v) .
$$

From this follows that

$$
X_{1} \times \complement \Gamma^{\circ} \subseteq \complement \mathrm{WF}_{\mathscr{F} \mathscr{B}}(v)
$$

Then Theorem 2.7 implies that $V \in \mathscr{F} \mathscr{B}$ at every point in $X_{1}+i\left(S^{d-1} \cap\right.$ $\left.\complement\left(-\Gamma^{\circ}\right)\right)$. Choose an open set $M$ with $\Gamma^{\circ} \cap S^{d-1} \subseteq M \subseteq S^{d-1}$ and where $\bar{M}$ belongs to the interior of $\Gamma^{\circ}$. Then $v=v_{1}+v_{2}$ where

$$
v_{1}=\int_{-\xi \notin M} V(\cdot+i \xi) d \xi
$$

belongs to $\mathscr{F} \mathscr{B}$ in $X_{1}$ and $v_{2}$ is the boundary value of the analytic function

$$
F(z)=\int_{-\xi \in M} V(z+i \xi) d \xi, \quad \operatorname{Im} z \in \Gamma_{1}, \quad|\operatorname{Im} z|<\gamma .
$$


Lemma 2.8 completes the proof.

As mentioned before we have that $\mathrm{WF}_{\mathscr{F} \mathscr{B}}(f) \subseteq \mathrm{WF}_{A}(f)$. In the following proposition we describe a relation between the wave-front sets of Fourier Banach function types and analytic wave-front sets.

Proposition 6.2. Let $\mathscr{B}$ be a translation invariant BF-space and $f \in$ $\mathscr{D}^{\prime}\left(\mathbf{R}^{d}\right)$. Then

$$
\mathrm{WF}_{\mathscr{F} \mathscr{B}}(f)=\bigcap_{g \in \mathscr{F} \mathscr{B}} \mathrm{WF}_{A}(f-g)
$$

For the proof we need the following Lemmas which are extensions of Proposition 1.5 and Lemma 1.6 in [15].

Lemma 6.3. Let $X \subseteq \mathbf{R}^{d}$ be open and $\mathscr{B}$ be a translation invariant BF-space. Then the map $\left(f_{1}, f_{2}\right) \mapsto f_{1} f_{2}$ from $\mathscr{S}\left(\mathbf{R}^{d}\right) \times \mathscr{S}\left(\mathbf{R}^{d}\right)$ to $\mathscr{S}\left(\mathbf{R}^{d}\right)$ extends uniquely to continuous mapping from $\mathscr{F} \mathscr{B}\left(\mathbf{R}^{d}\right) \times$ $\mathscr{F} L_{(v)}^{1}\left(\mathbf{R}^{d}\right)$ to $\mathscr{F} \mathscr{B}\left(\mathbf{R}^{d}\right)$.

Proof. (1) Let $f_{1} \in \mathscr{F} \mathscr{B}\left(\mathbf{R}^{d}\right)$ and $f_{2} \in \mathscr{S}\left(\mathbf{R}^{d}\right)$. By Minkowski's inequality it follows that

$$
\left\|f_{1} f_{2}\right\|_{\mathscr{F} \mathscr{B}}=\left\|\widehat{f}_{1} * \widehat{f}_{2}\right\|_{\mathscr{B}} \leq C\left\|\widehat{f}_{1}\right\|_{\mathscr{B}}\left\|\widehat{f}_{2}\right\|_{L_{(v)}^{1}} .
$$

The assertion (1) now follows from this estimate and the fact that $\mathscr{S}\left(\mathbf{R}^{d}\right)$ is dense in $\mathscr{F} L_{(v)}^{1}$.

Lemma 6.4. Let $X \subseteq \mathbf{R}^{d}$ be open, $f \in \mathscr{D}^{\prime}(X)$ and let $\mathscr{B}$ be a translation invariant BF-space. Also let $\left(x_{0}, \xi_{0}\right) \in X \times \mathbf{R}^{d} \backslash 0$. Then the following conditions are equivalent:

(1) $\left(x_{0}, \xi_{0}\right) \notin \mathrm{WF}_{\mathscr{F} \mathscr{B}}(f)$;

(2) there exists $g \in \mathscr{F} \mathscr{B}\left(\mathbf{R}^{d}\right)\left(g \in \mathscr{F} \mathscr{B}_{\text {loc }}(X)\right)$ such that $\left(x_{0}, \xi_{0}\right) \notin$ $\mathrm{WF}(f-g)$;

(3) there exists $g \in \mathscr{F} \mathscr{B}\left(\mathbf{R}^{d}\right)\left(g \in \mathscr{F}_{\mathscr{B}_{\text {loc }}}(X)\right)$ such that $\left(x_{0}, \xi_{0}\right) \notin$ $\mathrm{WF}_{A}(f-g)$.

Proof. In this proof we use the same ideas as in [13, Proposition 8.2.6] (see also [15] and [16]). We may assume that $g \in \mathscr{F}_{\text {Boc }}(X)$ in $(2)$ and (3) since the wave-front sets concern local properties. Assume that (2) holds. We can then find an open subset $X_{0}$ of $X$ and some open cone $\Gamma=\Gamma_{\xi_{0}}$ and a sequence $\varphi_{N} \in C_{0}^{\infty}$ such the $\varphi_{N}(f-g)=f-g$ on $X_{0}$ and

$$
\left|\mathscr{F}\left(\varphi_{N}(f-g)\right)(\xi)\right| \leq C_{N, \varphi_{N}}\langle\xi\rangle^{-N}, \quad N=1,2, \ldots, \xi \in \Gamma .
$$

In particular it follows that if $N_{0}$ is chosen large enough, then $\mid \varphi_{N}(f-$ $g) \mid \mathscr{F} \mathscr{B}(\Gamma)_{1}$ is finite for every $N>N_{0}$. Since $g \in \mathscr{F} \mathscr{B}\left(\mathbf{R}^{d}\right)$, it follows by Lemma 6.3 that $\left|\varphi_{N} g\right|_{\mathscr{F} \mathscr{B}(\Gamma)}$ is finite for every $\varphi_{N}$. Then $\left|\varphi_{N} f\right|_{\mathscr{F} \mathscr{B}(\Gamma)}$ is finite for every $N>N_{0}$ and (1) holds. 
Conversely, if $\left(x_{0}, \xi_{0}\right) \notin \mathrm{WF}_{\mathscr{F} \mathscr{B}}(f)$, then there exist an open neighbourhood $X_{0}$ of $x_{0}$ and an open conical neighbourhood $\Gamma$ of $\xi_{0}$ such that

$$
|\varphi f|_{\mathscr{F} \mathscr{B}(\Gamma)}<\infty
$$

when $\varphi \in C_{0}^{\infty}$, in view of Theorem 3.2 in [1].

Let $\varphi_{1}, \varphi \in C_{0}^{\infty}\left(X_{0}\right)$ be chosen such that $\varphi\left(x_{0}\right) \neq 0$ and $\varphi_{1}=1$ in the support of $\varphi$. Furthermore let $\widehat{g}=\mathscr{F}\left(\varphi_{1} f\right)$ in $\Gamma$ and otherwise 0 . Then $g \in \mathscr{F} \mathscr{B}\left(\mathbf{R}^{d}\right)$.

By [12, Lemma 8.1.1] and its proof, it follows that

$$
\left|\mathscr{F}\left(\varphi_{1}(\varphi f-g)\right)(\xi)\right|<C_{N}\langle\xi\rangle^{-N}, \quad N=0,1,2 \cdots,
$$

when $\xi \in \Gamma$ and $\Gamma$ is chosen sufficiently small. Since $\varphi \varphi_{1}=\varphi$ we have that (6.1) holds. This implies that $\left(x_{0}, \xi_{0}\right) \notin \mathrm{WF}(f-g)$. This proves that (1) and (2) are equivalent.

Since $\mathrm{WF}(f) \subseteq \mathrm{WF}_{A}(f)$ for each distribution $f$, it follows that (2) holds if (3) is fulfilled. Assume that (2) holds. Then in view of of the remark before Corollary 8.4.16 in [12] there exists some $h \in C^{\infty}(X)$ such that $\left(x_{0}, \xi_{0}\right) \notin \mathrm{WF}_{A}(f-g-h)$. Since $C^{\infty} \subseteq \mathscr{F}_{\mathscr{B}_{\text {loc }}}(X)$ it follows that $g_{1}=g+h \in \mathscr{F} \mathscr{B}_{\text {loc }}(X)$. Hence (3) holds, and the result follows.

Proof of Proposition 6.2. We start by showing that

$$
\mathrm{WF}_{\mathscr{F} \mathscr{B}}(f) \subseteq \mathrm{WF}_{A}(f-g),
$$

for every $g \in \mathscr{F} \mathscr{B}$. Since $\mathrm{WF}_{\mathscr{F} \mathscr{B}}(f-g) \subseteq \mathrm{WF}_{A}(f-g)$ it is sufficient to show that

$$
\mathrm{WF}_{\mathscr{F} \mathscr{B}}(f) \subseteq \mathrm{WF}_{\mathscr{F} \mathscr{B}}(f-g),
$$

for every $g \in \mathscr{F} \mathscr{B}$.

Assume that $\left(x_{0}, \xi_{0}\right) \notin \mathrm{WF}_{\mathscr{F} \mathscr{B}}(f-g)$. Then there exist $\varphi_{x_{0}} \in C_{0}^{\infty}$ with $\varphi_{x_{0}}\left(x_{0}\right) \neq 0$ and an open conical neighbourhood $\Gamma=\Gamma_{\xi_{0}}$ of $\xi_{0}$ such that

$$
\left\|\mathscr{F}\left(\varphi_{x_{0}}(f-g)\right) \chi_{\Gamma_{\xi_{0}}}\right\|_{\mathscr{B}}<\infty .
$$

It follows by Lemma 6.3 that

$$
\left\|\mathscr{F}\left(\varphi_{x_{0}} g\right) \chi_{\Gamma_{\xi_{0}}}\right\|_{\mathscr{B}}<\infty
$$

for every $g \in \mathscr{F} \mathscr{B}$ and then

$$
\left\|\mathscr{F}\left(\varphi_{x_{0}} f\right) \chi_{\Gamma_{\xi_{0}}}\right\|_{\mathscr{B}}=\left\|\mathscr{F}\left(\varphi_{x_{0}}(f-g)\right) \chi_{\Gamma_{\xi_{0}}}\right\|_{\mathscr{B}}+\left\|\mathscr{F}\left(\varphi_{x_{0}} g\right) \chi_{\Gamma_{\xi_{0}}}\right\|_{\mathscr{B}}<\infty .
$$

This shows that (6.2) holds. In fact, by similar calculations we can show the opposite inclusion and thereby obtain equality in (6.2). We have now shown that

$$
\mathrm{WF}_{\mathscr{F} \mathscr{B}}(f) \subseteq \bigcap_{g \in \mathscr{F} \mathscr{B}} \mathrm{WF}_{A}(f-g)
$$

We obtain the opposite inclusion by using Proposition 6.4. This completes the proof. 
Corollary 6.5. If $f \in \mathscr{D}^{\prime}(X)$ where $X$ is an interval on $\mathbf{R}$ and if $x_{0} \in X$ is a boundary point of $\operatorname{supp} f$, then $\left(x_{0}, \pm 1\right) \in \mathrm{WF}_{\mathscr{F} \mathscr{B}}(f)$.

Proof. Assume for example that $\left(x_{0},-1\right) \notin \mathrm{WF}_{\mathscr{F} \mathscr{B}}(f)$. Then we can find $F$ analytic in $\Omega=\left\{z ; \operatorname{Im} z>0,\left|z-x_{0}\right|<r\right\}$ with boundary value $f$. There is an interval $I \subseteq\left(x_{0}-r, x_{0}+r\right)$ where $f=0$. By Theorem 3.1.12 and 4.4.1 in Hörmander [12] $F$ can be extended analytically across $I$ so that $F=0$ below $I$. Thus the uniqueness of analytic continuation gives $F=0$, hence $f=0$ in $\left(x_{0}-r, x_{0}+r\right)$. This contradicts that $x_{0}$ is a boundary point of $\operatorname{supp} f$ and proves the corollary.

The result in the following lemma follows directly from Lemma 8.4.17 in Hörmander.

Lemma 6.6. If $f \in \mathscr{S}^{\prime}$ then $\mathrm{WF}_{\mathscr{F} \mathscr{B}}(f) \subseteq \mathbf{R}^{d} \times F$ where $F$ is the limit cone of supp $\widehat{f}$ at infinity, consisting of all limits of sequences $t_{j} x_{j}$ with $x_{j} \in \operatorname{supp} \widehat{f}$ and $0<t_{j} \rightarrow 0$.

Next we give some computational rules for wave-front sets of Fourier Banach function types. We prove that some of the rules that Hörmander obtained for classical and analytical wave-front sets in [12] holds also for wave-front sets of Fourier Banach function types. For completeness we give the proofs which are similar to those of the analogous results in $[12$.

Theorem 6.7. Let $X \subseteq \mathbf{R}^{d_{1}}$ and $Y \subseteq \mathbf{R}^{d_{2}}$ be open. Also let $f: X \rightarrow Y$ be a real analytic map with normal set $N_{f}$. Then

$$
\mathrm{WF}_{\mathscr{F} \mathscr{B}}\left(f^{*} g\right) \subseteq f^{*} \mathrm{WF}_{\mathscr{F} \mathscr{B}}(g), \quad \text { if } \quad g \in \mathscr{D}^{\prime}(Y), \quad N_{f} \cap \mathrm{WF}_{\mathscr{F} \mathscr{B}}(g)=\emptyset
$$

Proof. Assume that there exists an analytic function $\Phi$ in

$$
\Omega=\left\{y^{\prime}+i y^{\prime \prime} ; y^{\prime} \in Y, y^{\prime \prime} \in \Gamma,\left|y^{\prime \prime}\right|<\gamma\right\},
$$

where $\Gamma$ is an open convex cone, such that

$$
\left|\Phi\left(y^{\prime}+i y^{\prime \prime}\right)\right| \leq C\left|y^{\prime \prime}\right|^{-N} \quad \text { in } \Omega, \quad g=\lim _{\Gamma \ni y \rightarrow 0} \Phi(\cdot+i y) .
$$

Let $\Gamma^{\circ}$ be the dual $\Gamma$. Then by the arguments in the proof of Theorem 8.5.1 in Hörmander [12] it follows that $\mathrm{WF}_{A}(g) \subseteq Y \times \Gamma^{\circ}$. If we assume that $x_{0} \in X$ and that ${ }^{\mathrm{t}} \mathrm{f}^{\prime}\left(x_{0}\right) \eta \neq 0, \eta \in \Gamma^{\circ} \backslash 0$, then ${ }^{\mathrm{t}} \mathrm{f}^{\prime}\left(x_{0}\right) \Gamma^{\circ}$ is a closed, convex cone and

$$
\left.\mathrm{WF}_{A}\left(f^{*} g\right)\right|_{x_{0}} \subseteq\left\{\left(x_{0},{ }^{\mathrm{t}} f^{\prime}\left(x_{0}\right) \eta\right) ; \eta \in \Gamma^{\circ} \backslash 0\right\} .
$$

Next by using Corollary 2.9 and Theorem 6.1 it follows that any distribution $g$ can be written as a finite sum $\sum g_{j}$ where each term either belongs to $\mathscr{F} \mathscr{B}$ in a neighbourhood of $f\left(x_{0}\right)$ of satisfies the hypotheses above with some $\Gamma_{j}$ such that $\Gamma_{j}^{\circ}$ is small and intersects $\left.\mathrm{WF}_{\mathscr{F} \mathscr{B}}(g)\right|_{f\left(x_{0}\right)}$. 
By the hypotheses ${ }^{\mathrm{t}} f^{\prime}\left(x_{0}\right) \eta \neq 0$ when $\left(f\left(x_{0}\right), \eta\right) \in \mathrm{WF}_{\mathscr{F} \mathscr{B}}(f)$. We then conclude that

$$
\left.\mathrm{WF}_{\mathscr{F} \mathscr{B}}\left(f^{*} g\right)\right|_{x_{0}} \subseteq\left\{\left(x_{0},{ }^{\mathrm{t}} f^{\prime}\left(x_{0}\right) \eta\right), \eta \in \bigcup \Gamma_{j}^{\circ}\right\} .
$$

This implies (6.3).

Theorem 6.8. Let $f \in \mathscr{E}^{\prime}\left(\mathbf{R}^{d}\right)$. Split the coordinates in $\mathbf{R}^{d}$ into two groups $x^{\prime}=\left(x_{1}, \ldots, x_{d_{1}}\right)$ and $x^{\prime \prime}=\left(x_{d_{1}+1}, \ldots, x_{d}\right)$, and set

$$
f_{1}\left(x^{\prime}\right)=\int f\left(x^{\prime}, x^{\prime \prime}\right) d x^{\prime \prime}
$$

Then

$$
\mathrm{WF}_{\mathscr{F} \mathscr{B}}\left(f_{1}\right) \subseteq\left\{\left(x^{\prime}, \xi^{\prime}\right) ;\left(x^{\prime}, x^{\prime \prime}, \xi^{\prime}, 0\right) \in \mathrm{WF}_{\mathscr{F} \mathscr{B}}(f) \quad \text { for some } x^{\prime \prime}\right\} .
$$

Proof. We use the same notation as in Theorem 2.7. Then

$$
\langle f, \phi \otimes \psi\rangle=\int_{|\omega|=1}\langle U(\cdot+i \omega), \phi \otimes \psi\rangle d \omega,
$$

for $\phi \in C_{0}^{\infty}\left(\mathbf{R}^{d_{1}}\right)$ and $\psi \in C_{0}^{\infty}\left(\mathbf{R}^{d-d_{1}}\right)$. Take $\psi\left(x^{\prime \prime}\right)=\chi\left(\delta x^{\prime \prime}\right)$ where $\chi=1$ in the unit ball and let $\delta \rightarrow 0$. $U$ is decreasing exponentially at infinity and therefore it follows that

$$
\left\langle f_{1}, \phi\right\rangle=\int_{|\omega|=1}\langle U(\cdot+i \omega), \phi \otimes 1\rangle d \omega=\int_{|\omega|=1}\left\langle U_{1}\left(\cdot+i \omega^{\prime}\right), \phi\right\rangle d \omega
$$

where

$U_{1}\left(z^{\prime}\right)=\int U\left(z^{\prime}, x^{\prime \prime}\right) d x^{\prime \prime}=\int U\left(z^{\prime}, x^{\prime \prime}+i y^{\prime \prime}\right) d x^{\prime \prime}, \quad\left|\operatorname{Im} z^{\prime}\right|^{2}+\left|y^{\prime \prime}\right|^{2}<1$

is an analytic when $\left|\operatorname{Im} z^{\prime}\right|<1$, which is bounded by $C\left(1-\left|\operatorname{Im} z^{\prime}\right|\right)^{-N}$. If $\left|\omega_{0}^{\prime}\right|<1$ and $\left(x^{\prime}, x^{\prime \prime}, \omega_{0}^{\prime}\right) \notin \mathrm{WF}_{\mathscr{F} \mathscr{B}}(f)$ for every $x^{\prime \prime} \in \mathbf{R}^{d-d_{1}}$, then $u_{1} \in$ $\mathscr{F} \mathscr{B}$ at $x^{\prime}-i \omega_{0}^{\prime}$. Hence Lemma 2.8 implies that $\left(x^{\prime}, \omega_{0}^{\prime}\right) \notin \mathrm{WF}_{\mathscr{F} \mathscr{B}}(f)$.

Theorem 6.9. Let $X \subseteq \mathbf{R}^{d_{1}}$ and $Y \subseteq \mathbf{R}^{d_{2}}$ be open sets and $K \in$ $\mathscr{D}^{\prime}(X \times Y)$ be a distribution such that the projection $\operatorname{supp} K \rightarrow X$ is proper. If $f \in \mathscr{F} \mathscr{B}(Y)$ then

$\mathrm{WF}_{\mathscr{F} \mathscr{B}}(\mathscr{K} f) \subseteq\left\{(x, \xi) ;(x, y, \xi, 0) \in \mathrm{WF}_{\mathscr{F} \mathscr{B}}(K)\right.$ for some $\left.y \in \operatorname{supp} f\right\}$.

Here $\mathscr{K}$ is the linear operator with kernel $K$.

Proof. Replace $K$ by $K(1 \otimes f)$ and assume that $f=1$. Without changing $K$ over a given compact subset of $X$ we may replace $K$ by a distribution of compact support, and then the statement is identical to Theorem 6.8. 


\section{REFERENCES}

[1] S. Coriasco, K. Johansson, J. Toft Wave-front sets of Banach function types, Preprint, in arXiv:0911.1867v1, 2009

[2] H. G. Feichtinger Modulation spaces on locally compact abelian groups. Technical report, University of Vienna, Vienna, 1983; also in: M. Krishna, R. Radha, S. Thangavelu (Eds) Wavelets and their applications, Allied Publishers Private Limited, NewDehli Mumbai Kolkata Chennai Hagpur Ahmedabad Bangalore Hyderbad Lucknow, 2003, pp. 99-140.

[3] Wiener amalgams over Euclidean spaces and some of their applications, in: Function spaces (Edwardsville, IL, 1990), Lect. Notes in pure and appl. math., 136, Marcel Dekker, New York, 1992, pp. 123Ü137.

[4] H. G. Feichtinger and K. H. Gröchenig Banach spaces related to integrable group representations and their atomic decompositions, I, J. Funct. Anal., 86 (1989), 307-340.

[5] B_Banach spaces related to integrable group representations and their atomic decompositions, II, Monatsh. Math., 108 (1989), 129-148.

[6] _ Gabor frames and time-frequency analysis of distributions, J. Functional Anal., 146 (1997), 464-495.

[7] _ Modulation spaces: Looking back and ahead, Sampl. Theory Signal Image Process. 5 (2006), 109-140.

[8] H. G. Feichtinger and K. H. Gröchenig, Banach spaces related to integrable group representations and their atomic decompositions. I, J. Funct. Anal., 86 (1989), 307-340.

[9] K. H. Gröchenig Describing functions: atomic decompositions versus frames, Monatsh. Math.,112 (1991), 1-42.

[10] K. Gröchenig, Foundations of Time-Frequency Analysis, Birkhäuser, Boston, 2001.

[11] K. Gröchenig, J. Toft Isomorphism properties of Toeplitz operators and pseudo-differential operators between modulation spaces, Preprint, in arXiv:0905.4954v2, 2009.

[12] L. Hörmander The Analysis of Linear Partial Differential Operators, vol I-III, Springer-Verlag, Berlin Heidelberg NewYork Tokyo, 1983, 1985.

[13] Lectures on Nonlinear Hyperbolic Differential Equations, SpringerVerlag, Berlin, 1997.

[14] S. Pilipović, N. Teofanov, J. Toft, Micro-local analysis in Fourier Lebesgue and modulation spaces. Part I, preprint, in arXiv:0804.1730v3, 2009.

[15] _ Micro-local analysis in Fourier Lebesgue and modulation spaces. Part $I I$, preprint, in arXiv:0805.4476v2, 2008.

[16] Wave-front sets in Fourier Lebesgue space, Rend. Sem. Mat. Univ. Politec. Torino, 66 (4) (2008), 41Ŭ61.

Department of Computer science, Physics and Mathematics, Linnæus University, Växjö, SwedeN1]

E-mail address: karoline.johansson@lnu.se

\footnotetext{
${ }^{1}$ Former address: Department of Mathematics and Systems Engineering, Växjö University, Sweden
} 